\title{
Multilane Traffic Flow Modeling Using Cellular Automata Theory
}

\author{
Antonina Chechina ${ }^{1, \star}$, Natalia Churbanova ${ }^{1, \star \star}$, and Marina Trapeznikova ${ }^{1, \star \star \star}$ \\ ${ }^{1}$ Keldysh Institute of Applied Mathematics RAS, 4 Miusskaya Square, Moscow, Russia
}

\begin{abstract}
The paper deals with the mathematical modeling of traffic flows on urban road networks using microscopic approach. The model is based on the cellular automata theory and presents a generalization of the Nagel-Schreckenberg model to a multilane case. The created program package allows to simulate traffic on various types of road fragments (T or X type intersection, strait road elements, etc.) and on road networks that consist of these elements. Besides that, it allows to predict the consequences of various decisions regarding road infrastructure changes, such as: number of lanes increasing/decreasing, putting new traffic lights into operation, building new roads, entrances/exits, road junctions.
\end{abstract}

\section{Introduction}

There are two main approaches to the traffic flow modeling: macroscopic, in which the vehicle flow is presented (in the dynamic case) as a compressible fluid flow, and microscopic, where each car is considered separately. The macroscopic approach is characterized by its simpicity - the computational complexity and the calculation time do not depend on the amount of cars in the network. It uses average characteristics of the traffic flow, such as the flow density and velocity. But there are serious restrictions: this approach can't be applied if the density is too low; also, the modeling can be carried out only on long (in comparison with car sizes) road fragments, which is not suitable for traffic simulation on crossroads and small road networks of city districts. The microscopic approach is free of these restrictions and can be applied to a flow with any density and on any road fragment. It also can provide much more detailed information about the traffic dynamics. It can accommodate a great many nuances of the real flows, such as the difference in driver's behavior and strategies, as well as the reactions on other drivers' actions, various car properties and so on. Modern computational facilities, such as high-performance supercomputer systems, allow real-time calculations on large-scale road networks, that is why microscopic approach becomes more and more popular nowadays.

\section{Two-dimensional microscopic model based on cellular automata theory and the Nagel-Schreckenberg model}

The model created by the present authors is based on the cellular automata theory: the road is divided into equal cells, each cell can either contain a vehicle, or be empty. A cell is $7.5 \mathrm{~m}$ long and one

\footnotetext{
$\star$ e-mail: chechina.antonina@yandex.ru

$\star \star$ e-mail: nataimamod@mail.ru

$\star \star \star$ e-mail: mtrapez@yandex.ru
} 
lane wide; the distance in the model is measured in the number of cells, speed of a vehicle - in the number of cells that a vehicle can cover during one time step, time - in time steps, one second long each. As all the cells are equal in size and can fit in only a light car, the model cannot represent heterogeneous traffic flows, that include heavy vehicles, such as lorries and trucks. As opposed to the classic Nagel-Schreckenberg model [1], the developed model is two-dimensional and allows to depict multilane traffic.

Each car has a set of parameters: unique ID, maximum speed, current speed and final destination. A road fragment with cars is shown in Fig. 1. The car color in the picture corresponds to different destination points (road exits, turns, etc.)

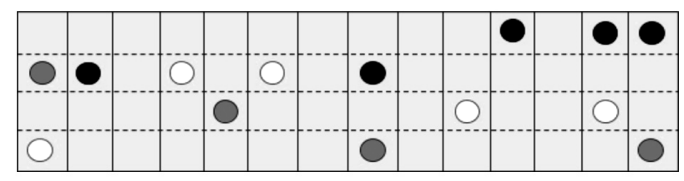

Figure 1. Multilane road fragment with cars

Each time step cell state update is carried out according to the following rules:

- a vehicle changes a lane if it is necessary (to reach the desired destination or to drive around an obstacle), it is advantageous for a driver (leads to speed increase and/or density decrease) and it is possible (i.e., if the lane change is allowed and the target cell is empty);

- a vehicle moves along the road according to the classic rules for the one-lane traffic.

For more details, see [2]. The works featuring the comparison between the created model and other approaches can be found in the list of references in [2].

\section{Modeling different driving strategies}

Each driver has his own driving style: ones are more determined, change lanes more easily, others are more cautious, they wait for a larger gap to move to the neighboring lane. Their behavior influences the overall situation on the road. To reflect that, the following driver types were included in the model:

- "Cautious" drivers change lanes only if the gap between the target cell and the first occupied cell upstream is larger than the maximum speed;

- "Aggressive" drivers change lanes if the gap between the target cell and the first occupied cell upstream is larger than the actual speed of the vehicle which is situated in the first occupied cell;

- "Cooperative" drivers can be either cautious or aggressive. The percentage of cooperative drivers in the system can vary. These drivers:

- slow down $(v=v-1, v \geq 1)$, if they see a traffic jam before an obstacle or a road bottleneck on the neighboring lane;

- if there is a jam before the obstacle on the neighboring lane, and there are drivers that want to change their lane, cooperative drivers stop and let them pass;

- wait for several time steps if the car from the other lane can't move immediately, because the target cell is occupied by another car. 


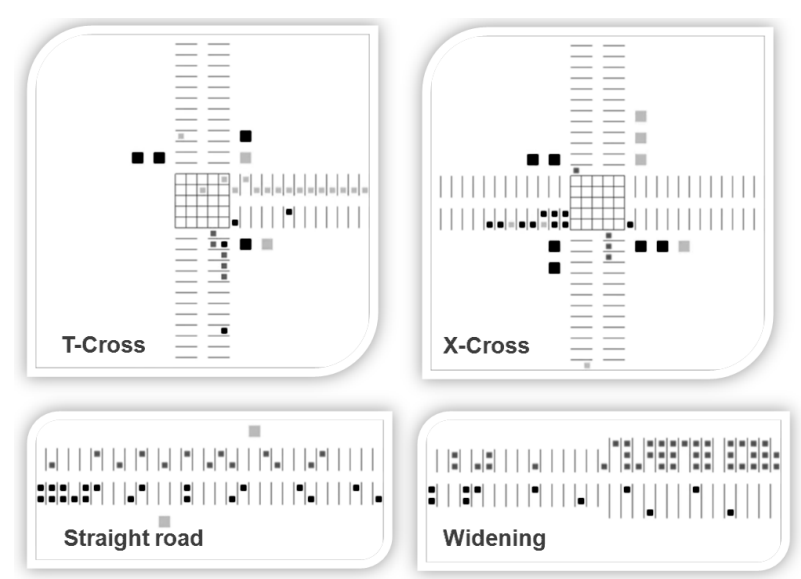

Figure 2. Simulation of traffic on different road elements

\section{Modeling traffic flows on road elements and small road networks}

The traffic flow modeling on different kinds of road elements can be executed using the created microscopic model. The numerical realization is represented as a module-structured program package implemented in $\mathrm{C}++$ and a the visualization block (based on the Glut library), which allows to see the step-by-step evolution of the system along with the computations. The result of the work of the package is shown in Fig. 2, featuring a T-crossroad and an X-crossroad with traffic lights, a straight road fragment with a traffic light for pedestrians, and a road fragment with a widening. The larger squares represent traffic lights (darker ones indicate red light, lighter ones - green light), the smaller squares point to cars with different final destinations. Special algorithms are developed for crossroad overcoming, widening/narrowing of the road, forming an entrance queue [2].

Being able to model traffic flows on different types of road elements, we can combine them, building various road networks. The computations for each element can be carried out in parallel with data exchange on the borders: cars leave one fragment and enter another one. The example of small road network that consists of two intersections: a T-cross and an X-cross, is shown in Fig. 3.

Different kinds of average characteristics (such as, for instance, the capacity of the crossroad) can be also obtained using the program package.

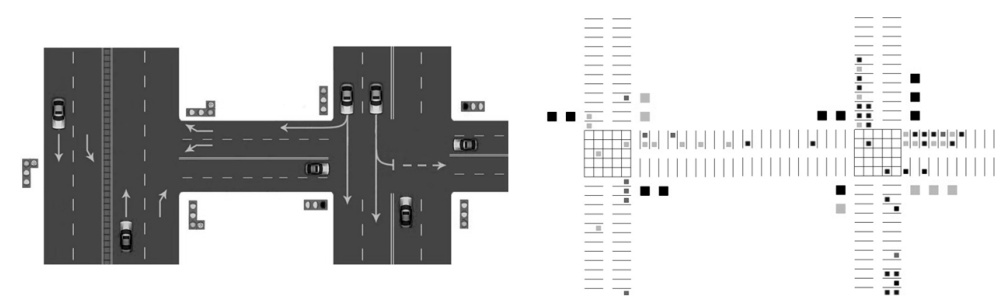

Figure 3. Road network: a scheme (on the left) and a simulation result (on the right) 


\section{Modeling a road accident}

Road accidents can not happen inside the model due to the safety rules: a car always slows down approaching an obstacle or another car in the lane, and it doesn't change its lane if the gap in the target lane is not enough to do it safely. But it is possible to model consequences of an accident (see Fig. 4), that might be necessary for real-time online modeling of city traffic in order to develop the measures to avoid traffic jams and evaluate the efficiency of these measures. It is also possible to evaluate how much the capacity of a road will decrease after the accident. In Fig. 4 the influence of driving strategies on the road capacity in case of the accident is shown. Black squares represent cars that stopped due to the accident, and grey squares - moving cars that have to drive around them. On the left picture all drivers in the system are "cautious" drivers, and on the right picture all drivers are "aggressive". As is seen from the pictures, there is a congestion before the obstacle in both cases, but the density of cars in case of "cautious" drivers is larger, this behavior decreases the road capacity more significantly and leads to a traffic jam faster than in the case of "aggressive" driving.

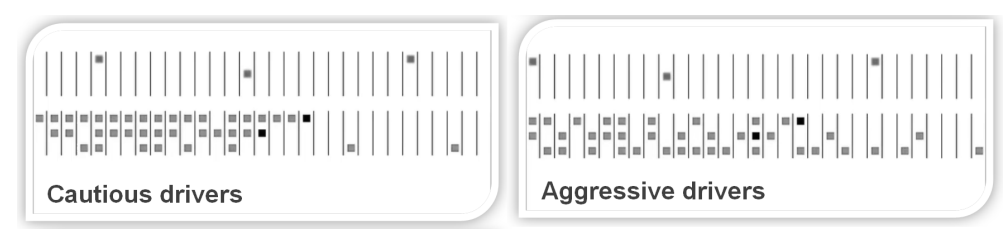

Figure 4. Road accident: influence of driving strategies ("cautious" or "aggressive") on jam formation

\section{Conclusions}

The model and algorithms developed showed results comparable with the simulation by the standard program software. The created program package can be used for traffic modeling on city road networks, taking into consideration different driving strategies. Due to the inner parallelism and the simplicity of the numerical algorithm the calculations can be carried out using high performance supercomputers on large-scale road networks in real time.

\section{Acknowledgment}

The work is supported by RFBR projects 16-31-00087, 16-01-00347, 18-01-00405.

\section{References}

[1] K. Nagel and M. Schreckenberg, J. Phys. I France 2, 2221 (1992)

[2] A. Chechina, N. Churbanova, and M. Trapeznikova, Traffic and Granular Flow'13 (M. Chraibi et al. Eds., Springer, 2015) 361-368 